\title{
Hereditary Motor and Sensory Neuropathy Type VI with Bilateral Middle Cerebellar Peduncle Involvement
}

\author{
Jung-Hwan $\mathrm{Oh}^{1}$, Han Sang Lee ${ }^{2}$, Dong Min $\mathrm{Cha}^{3}$ and Sa-Yoon Kang ${ }^{1 *}$ \\ ${ }^{1}$ Department of Neurology, JeJu National University Hospital, JeJu 690-767, ${ }^{2}$ Department of Neurology, Seoul National \\ University Hospital, Seoul 110-744, ${ }^{3}$ Department of Ophthalmology, JeJu National University Hospital, JeJu 690-767, Korea
}

Charcot-Marie-Tooth disease (CMT) 2A with optic atrophy is referred to as hereditary motor and sensory neuropathy type VI (HMSN VI) and is caused by mitofusin 2 gene (MFN2) mutation. In patients with MFN2 related CMT, central nervous system is known to be also involved and cerebral white matter is mostly involved. We report a patient confirmed as HMSN VI who had isolated bilateral middle cerebellar peduncular lesions in brain MRI.

Key words: hereditary motor and sensory neuropathy, magnetic resonance imaging, mitofusin, mitochondria

\section{INTRODUCTION}

Hereditary neuropathies are mainly divided into three subcategories: hereditary motor and sensory neuropathy (HMSN), hereditary sensory neuropathy (HSN), and hereditary motor neuropathy (HMN) [1], and these disorders are related with mutations in about 50 genes [2]. Charcot-Marie-Tooth disease (CMT), also known as HMSN, is one of the most common inherited neuropathies, affecting approximately 1 in 2,500 people [1]. Most forms of CMT are inherited in an autosomal dominant fashion. CMTs are traditionally divided into two types: demyelinating neuropathy or abnormal myelination, also called HMSN-I (CMT1), and axonal neuropathy, also called HMSNII (CMT2) [2]. Symptoms of CMT vary including peripheral

Received August 10, 2014, Revised August 28, 2014,

Accepted August 30, 2014

* To whom correspondence should be addressed. TEL: 82-64-717-1620, FAX: 82-64-757-8276 e-mail: neurokang@jejunu.ac.kr neuropathy symptoms, optic nerve atrophy, spastic paraparesis, retinitis pigmentosa and others [1,2]. HMSN VI is an axonal CMT $2 \mathrm{~A}$ with optic atrophy [1]. HMSN VI is caused by mutations in mitofusin 2 (MFN2). The MFN2 protein participates in regulation of mitochondria metabolism [2]. Since the central nervous system has a large potential oxidative capacity due to the high level of oxygen consumption [3], involvement of the central nervous system is an unsurprising event in patients with CMT associated with MFN2 mutation. According to a recent study of Chung et al. [4] which has a relatively large number of patients with MFN2 related CMT, cerebral involvements are detected in 38\% (8 of 21) of all subjects, and most patients have white-matter lesions on brain MRI scanning. The isolated involvement of bilateral middle cerebellar peduncles has been very rarely reported in patients with HMSN VI, therefore, we report this rare case with a brief review of literature.

\section{CASE REPORT}

A 47-year old woman visited our hospital with a chief complaint
Copyright $\odot$ Experimental Neurobiology 2014. www.enjournal.org
This is an Open Access article distributed under the terms of the Creative Commons Attribution Non-Commercial License (http://creativecommons.org/licenses/by-nc/3.0) which permits unrestricted non-commercial use, distribution, and reproduction in any medium, provided the original work is properly cited. 


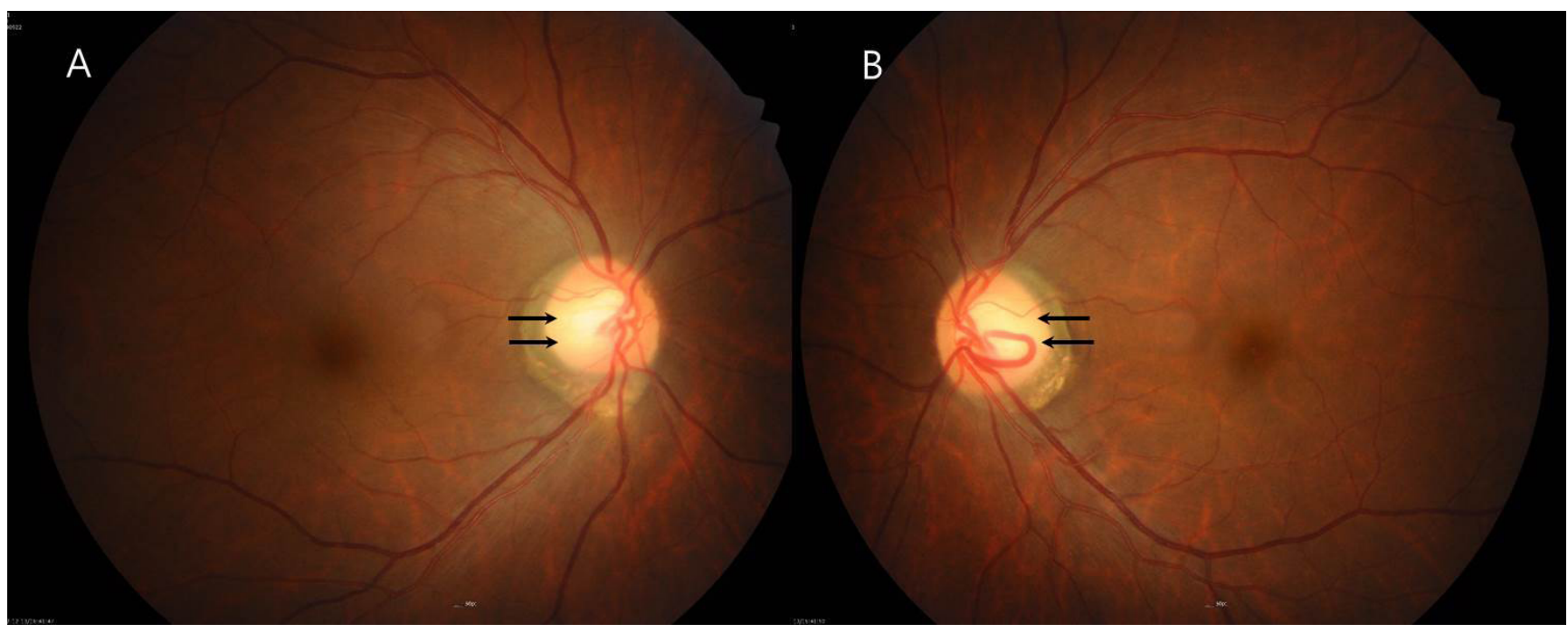

Fig. 1. Bilateral mild optic disc pallor of the temporal sides (arrows) in color fundus photogram (A, right side; B, left side).

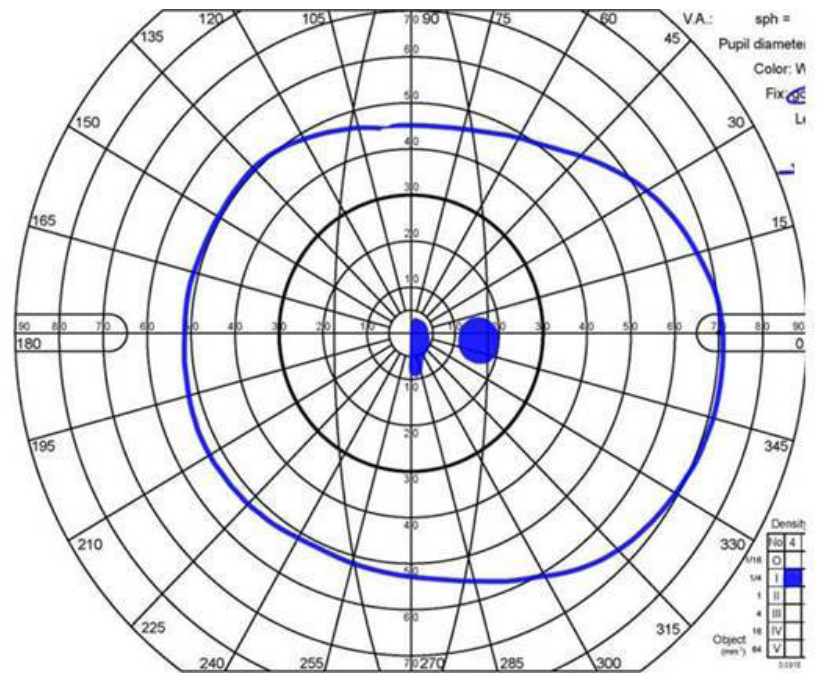

Fig. 2. Goldmann perimetry showed bilateral central scotomas.

of visual impairment in both eyes. The patient had muscle weakness and atrophy in distal lower extremities from about 7 years old, and used a wheelchair due to a disability that makes ambulation impossible as symptoms deteriorated. Muscle weakness and atrophy in both hands developed from about 20 years old. Her symptoms gradually deteriorated and the affected area expanded to the entire upper extremities. Four months before her visit to our hospital, visual acuity in both eyes had gradually reduced. Recently, she had a difficulty in recognizing people's faces from a $5 \mathrm{~m}$ distance, but had no color vision deficiency or ocular pain. She had normal cognitive function development and achieved outstanding academic grades. After finishing graduate school, she is currently working as a teacher.

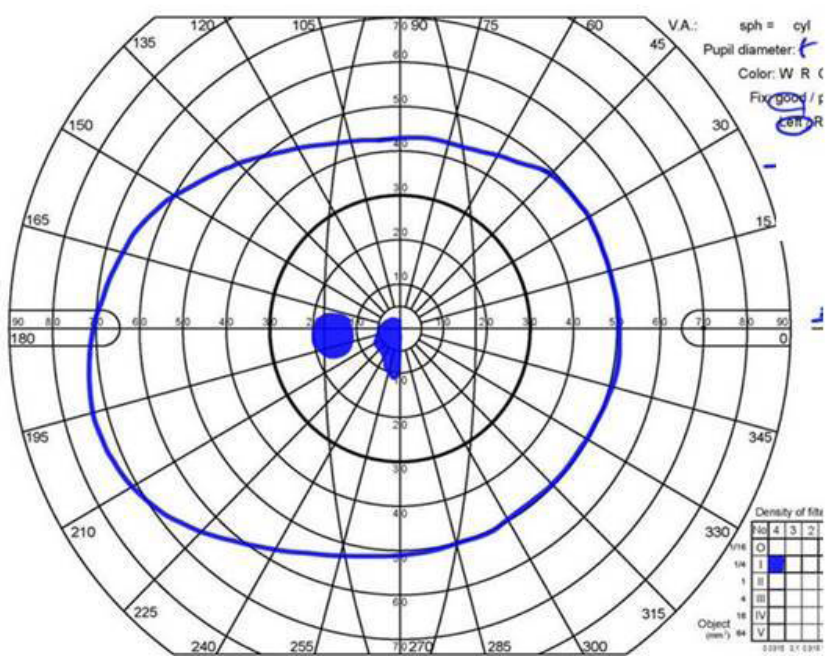

Physical examination revealed that the patient had normal cognitive function, and her pupils were $3 \mathrm{~mm} / 3 \mathrm{~mm}$, symmetric and reactive to light. According to ophthalmologic examination, corrected visual acuity was 20/130 in the right eye and 20/200 in the left eye, and uncorrected visual acuity was 20/500 in the right eye and 20/1,000 in the left eye. Although the retina was normal in all eyes on slit lamp examination, bilateral optic disc pallor was detected on the temporal sides in fundus photogram (Fig. 1). Visual field constriction was not observed, but central scotoma in both eyes was found on Goldmann perimetry (Fig. 2). She had no motor disturbances in the extraocular and facial muscles. Dysarthria and dysphagia were not detected. Diffuse muscle weakness and atrophy were observed in both upper and lower 


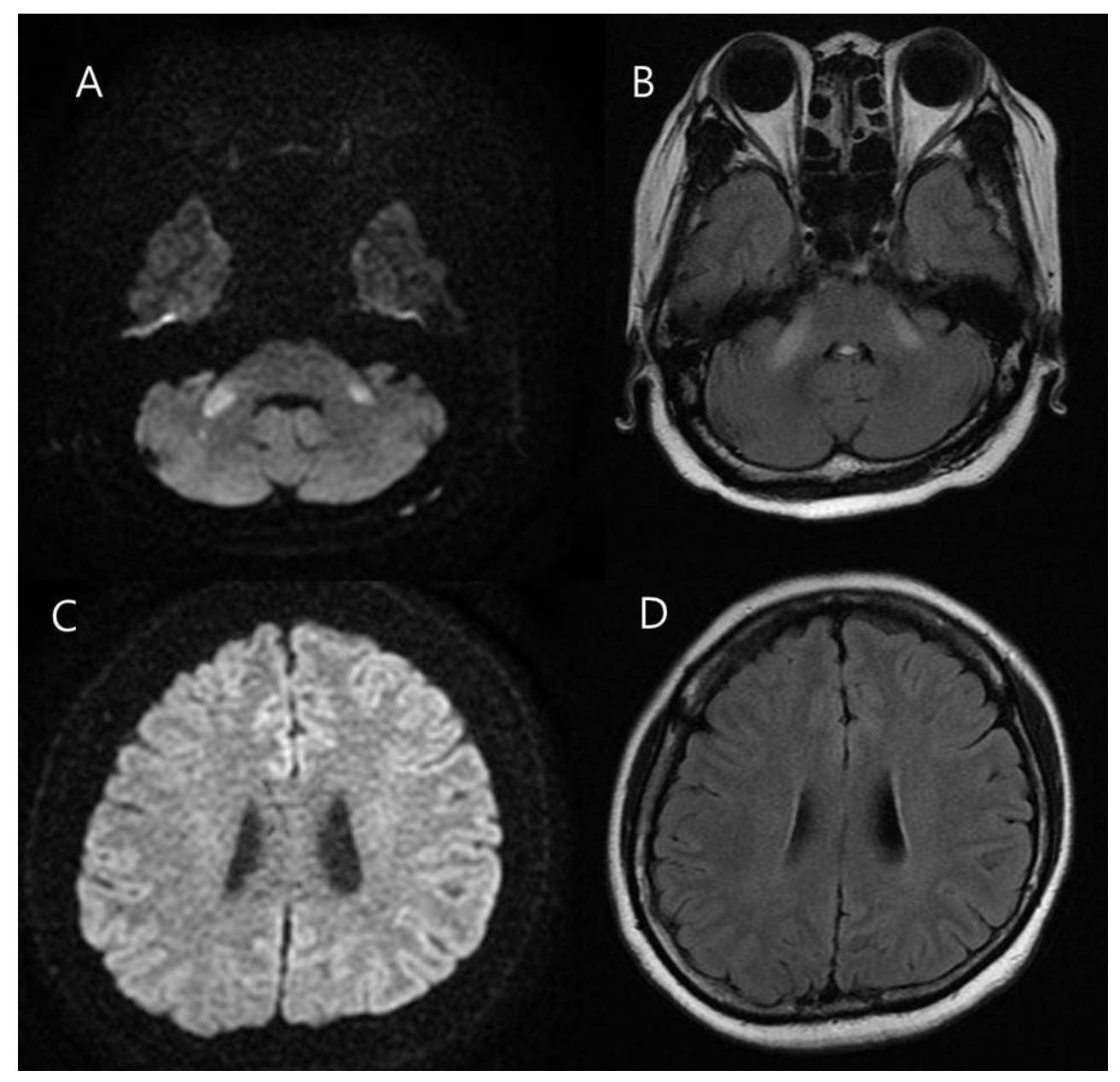

Fig. 3. Diffusion (A and C) and fluid attenuated inversion recovery (FLAIR) MRI (B and D) at admission day 1 . High signal intensities of bilateral middle cerebellar peduncles in diffusion and FLAIR MRI (A and B) were noted. However, there is no abnormal signal change in cerebral white matter $(\mathrm{C}$ and D). extremities, and the symptoms were more severe in the distal extremities. The sensory function was normal, and tendon reflexes were absent in both upper and lower extremities. Babinski reflexes were negative.

There were no electric pontentials in sensory and motor nerve conduction studies and needle electromyographic examinations revealed giant motor unit action potentials and reduced interference patterns. Pattern reversal visual evoked potentials were significantly prolonged in both eyes. MRI scanning of the cerebral hemispheres, orbits and optic nerves was normal, but high signal intensities were found in bilateral middle cerebellar peduncles on diffusion-weighted and fluid attenuated inversion recovery (FLAIR) imaging (Fig. 3). Her routine blood laboratory studies were normal, and serum lactate level was also normal at 2.5 $\mathrm{mmol} / \mathrm{L}$. She is the eldest daughter of a son and five daughters. No family members had symptoms of neuromuscular disorders.

We analyzed exons 1 19 of MFN2 and their franks region by direct cycle sequencing, and identified heterozygous transversion of cytosine to thymine $(\mathrm{c} .617 \mathrm{C}>\mathrm{T})$ in the seventh coding exon, when compared with the reference sequences(NC_000001.10, NM_014874.3). This mutation has already been reported in CMT [1]. Mitochondrial DNA mutations associated with Leber's hereditary optic atrophy $(3460 \mathrm{G}>\mathrm{A}, 11778 \mathrm{G}>\mathrm{A}, 14484 \mathrm{~T}>\mathrm{C}$ and $4171 \mathrm{C}>\mathrm{A}$ ) were not found. Because the patient had not only peripheral neuropathy but also optic atrophy, she was diagnosed with HMSN VI caused by MFN2 mutation.

\section{DISCUSSION}

Nicholson et al. [5] first identified MFN2 mutations on chromosome 1p 36 in patients with HMSN VI, and mutations in MFN2 gene were also related with CMT2A and CMT5. MFN2 is a mitochondrial outer membrane GTPase and is related with fusion and fission of mitochondria [2]. OPA1 (optic atrophy 1), located in the inner mitochondrial membrane, is associated with the most common type of pure autosomal dominant optic atrophy [1]. Since MFN2 and OPA1 have complimentary functions of mitochondrial dynamics, MFN2 mutation is able to cause optic 
neuropathy as well as peripheral neuropathy [2].

MFN2 related CMT tends to involve the periventricular white matter rather than the cerebral cortex in the central nervous system $[4,6]$. This may be relevant to MFN2 functions. MFN2 also plays a role in mitochondrial trafficking along the neurons through the microtubule [2]. Baloh et al. have asserted that the distal axons far from cell bodies of neurons are inclined to have an altered energy metabolism in the MFN2 mutant peripheral neurons, because the mitochondria supplement was insufficient in the region of the axons [7]. Interestingly, the patient had lesions only in the bilateral middle cerebellar peduncles on brain MRI scanning. The middle cerebellar peduncles are paired structures that connect the cerebellum with axons of long motor and sensory neurons arising from the cerebral cortex through pontine nuclei. Therefore, the lesions are likely to occur only in middle cerebellar peduncles because of insufficient mitochondrial trafficking compared to the cerebral white matter, which is composed of relatively shorter axons from cell bodies. Moreover, bilateral middle cerebellar peduncles were altered, mostly due to the neurodegenerative disease [8]. Since mitochondrial dysfunction is a substantially significant cause for neurodegenerative diseases [3], MFN 2 related diseases such as HMSN VI seem to be profoundly associated with degeneration of the middle cerebellar peduncle. Although a previous study reported the only case of involvement of the middle cerebellar peduncles in a patient with HMSN VI [1], brain MRI images were not provided. In the case, MFN2 mutation site was c.1252C>T (p.R418X), unlike the patient in our case.

Although MFN2 is ubiquitously expressed in the whole nervous system [2], it does not explain why involvement of the central nervous system is relatively rare compared to that of the peripheral nervous system in patients with MFN2 related CMT. The relationship between abnormal mitochondrial function caused by MFN2 mutation and involvement of the central nervous system still remains unclear. For this reason, additional studies are required and brain imaging techniques including MRI will be beneficial in clarifying the pathogenic mechanism, in addition to providing diagnostic clues, in patients with hereditary motor and sensory neuropathy.

\section{REFERENCES}

1. Züchner S, De Jonghe P, Jordanova A, Claeys KG, Guergueltcheva V, Cherninkova S, Hamilton SR, Van Stavern G, Krajewski KM, Stajich J, Tournev I, Verhoeven K, Langerhorst CT, de Visser M, Baas F, Bird T, Timmerman V, Shy M, Vance JM (2006) Axonal neuropathy with optic atrophy is caused by mutations in mitofusin 2. Ann Neurol 59:276-281.

2. Pareyson D, Piscosquito G, Moroni I, Salsano E, Zeviani M (2013) Peripheral neuropathy in mitochondrial disorders. Lancet Neurol 12:1011-1024.

3. Calabrese V, Scapagnini G, Giuffrida Stella AM, Bates TE, Clark JB (2001) Mitochondrial involvement in brain function and dysfunction: relevance to aging, neurodegenerative disorders and longevity. Neurochem Res 26:739-764.

4. Chung KW, Kim SB, Park KD, Choi KG, Lee JH, Eun HW, Suh JS, Hwang JH, Kim WK, Seo BC, Kim SH, Son IH, Kim SM, Sunwoo IN, Choi BO (2006) Early onset severe and lateonset mild Charcot-Marie-Tooth disease with mitofusin 2 (MFN2) mutations. Brain 129:2103-2118.

5. Nicholson GA, Valentijn LJ, Cherryson AK, Kennerson ML, Bragg TL, DeKroon RM, Ross DA, Pollard JD, McLeod JG, Bolhuis PA, Baas F (1994) A frame shift mutation in the PMP22 gene in hereditary neuropathy with liability to pressure palsies. Nat Genet 6:263-266.

6. Brockmann K, Dreha-Kulaczewski S, Dechent P, Bönnemann C, Helms G, Kyllerman M, Brück W, Frahm J, Huehne K, Gärtner J, Rautenstrauss B (2008) Cerebral involvement in axonal Charcot-Marie-Tooth neuropathy caused by mitofusin2 mutations. J Neurol 255:1049-1058.

7. Baloh RH, Schmidt RE, Pestronk A, Milbrandt J (2007) Altered axonal mitochondrial transport in the pathogenesis of Charcot-Marie-Tooth disease from mitofusin 2 mutations. J Neurosci 27:422-430.

8. Okamoto K, Tokiguchi S, Furusawa T, Ishikawa K, Quardery AF, Shinbo S, Sasai K (2003) MR features of diseases involving bilateral middle cerebellar peduncles. AJNR Am J Neuroradiol 24:1946-1954. 